\title{
On Uniqueness of New Orthogonality via 2-HH Norm in Normed Linear Space
}

\author{
Bhuwan Prasad Ojha $\mathbb{D}^{1},{ }^{1}$ Prakash Muni Bajracharya $\mathbb{D}^{1},{ }^{1}$ and Vishnu Narayan Mishra $\mathbb{D}^{2}$ \\ ${ }^{1}$ Central Department of Mathematics, Tribhuvan University, Kirtipur, Kathmandu, Nepal \\ ${ }^{2}$ Department of Mathematics, Faculty of Science, Indira Gandhi National Tribal University, Lalpur, Amarkantak, Anuppur, \\ Madhya Pradesh, India \\ Correspondence should be addressed to Bhuwan Prasad Ojha; bhuwanp.ojha@apexcollege.edu.np
}

Received 29 August 2020; Revised 23 October 2020; Accepted 2 November 2020; Published 21 November 2020

Academic Editor: Syed Abdul Mohiuddine

Copyright (c) 2020 Bhuwan Prasad Ojha et al. This is an open access article distributed under the Creative Commons Attribution License, which permits unrestricted use, distribution, and reproduction in any medium, provided the original work is properly cited.

\begin{abstract}
This paper generalizes the special case of the Carlsson orthogonality in terms of the 2-HH norm in real normed linear space. Dragomir and Kikianty (2010) proved in their paper that the Pythagorean orthogonality is unique in any normed linear space, and isosceles orthogonality is unique if and only if the space is strictly convex. This paper deals with the complete proof of the uniqueness of the new orthogonality through the medium of the 2- $\mathrm{HH}$ norm. We also proved that the Birkhoff and Robert orthogonality via the $2-\mathrm{HH}$ norm are equivalent, whenever the underlying space is a real inner-product space.
\end{abstract}

\section{Introduction}

Different notions of orthogonality in normed linear spaces have been developed by various mathematicians. As a generalization of orthogonality from inner product space to normed linear space " $x$ is orthogonal to $y$ if and only if $\|x+\lambda y\|=\|x-\lambda y\|$ identically in $\lambda$ " was suggested by Robert $([1,2])$. However, it has the weakness that for some normed linear space, at least one of every pair of orthogonal elements would have to be zero, i.e., $\|x+\lambda y\|$ $=\|x-\lambda y\|$ for all $\lambda$ only if $x=0$ or $y=0$. This difficulty is not experienced in the isosceles, Pythagorean, and Birkhoff orthogonalities.

To study the difference of orthogonality in the complex case in comparison with the real case, Paul et al. in 2018 came with a new concept of Birkhoff-James orthogonality by introducing new definitions on complex reflexive Banach spaces and introduced more than one equivalent characterization of Birkhoff-James orthogonality of compact linear operators in the complex case [3]. In 1945, James came with the concept of the Pythagorean and isosceles orthogonalities, which characterize inner product space via their homogeneity and additivity [4]. James also discussed the existence property of isosceles orthogonality type. The property of the uniqueness of isosceles orthogonality was not discussed until Kapoor and Prasad's paper was published. They proved that the Pythagorean orthogonality is unique in any normed linear space; however, the isosceles orthogonality is unique if and only if the space is strictly convex [5].

Carlsson introduced a more general type of orthogonality treating the isosceles and Pythagorean orthogonalities are special cases [6]. Martini and Wu showed many interesting connections between the Birkhoff and isosceles orthogonality. They proved that if a linear map preserves the Birkhoff orthogonality, then it also preserves the isosceles orthogonality [7]. In 2007, Alsina and Tomas gave a different characterization of the inner product space with the help of weaker linearity axioms of the scalar product and Pythagoras/isosceles orthogonality [8].

Using the concept of the p-HH norm as described in the paper [9], Kikianty and Dragomir came up with a new notion of orthogonality with the help of the 2-HH norm, which is closely related to the Pythagorean and isosceles orthogonalities [10]. They proved that the Pythagorean orthogonality via $2-\mathrm{HH}$ norm satisfies the nondegeneracy, continuity, and symmetry properties; however, it is neither additive nor 
homogeneous in normed linear space, but it satisfies the property of existence and uniqueness in any normed linear spaces. Isosceles orthogonality via the $2-\mathrm{HH}$ norm also satisfies the nondegeneracy, continuity, and symmetry properties but neither additive nor homogeneous in general. If the normed linear space $X$ is convex, then the isosceles orthogonality via $2-\mathrm{HH}$ norm satisfies the property of uniqueness, but the existent property holds in any normed linear space [10].

According to Carlsson's result described in [6], the isosceles and Pythagorean orthogonalities are special cases of the generalized Carlsson orthogonality. We introduced a new special case of the Carlsson orthogonality which satisfies all requirements as stated in Carlsson's orthogonality as well as the nondegeneracy, simplification, and continuity property of the inner product space. Furthermore, we proved that such orthogonality is homogeneous if and only if the underlying space is an inner product space [11]. Motivated by the results of Kikianty and Dragomir, and our previous result, we have attempted to introduce a new notion of orthogonality through the medium of the 2-HH norm, which we denote by the $2-\mathrm{HH}-\mathrm{N}$ orthogonality. We have proved that the 2$\mathrm{HH}-\mathrm{N}$ orthogonality is unique in any normed linear space. If the norm on $X$ is induced by an inner product, then the Robert and Birkhoff orthogonality via the $2-\mathrm{HH}$ norm is equivalent.

\section{Definition Notation and Preliminary Results}

Let us first establish the notations and terminologies used in this paper. Let $X$ be the normed linear space which we consider to be real. For any $x, y \in X, 2-\mathrm{HH}-\mathrm{N}$ denotes $x$ as the orthogonal to $y$ via the $2-\mathrm{HH}$ norm, which we defined with the help of the new special case of the Carlsson orthogonality discussed in [11]. The Pythagorean orthogonality plays an important role in describing new orthogonality through the medium of the 2-HH norm. Given any two elements $x, y \in$ $X$, we say that $x$ is Pythagorean orthogonal to $y$, written as $x \perp_{P} y$, if and only if $\|x+y\|^{2}=\|x\|^{2}+\|y\|^{2}$ [4]. Kikianty and Dragomir introduced the Pythagorean orthogonality via the 2-HH norm and using a similar idea to that of Kapoor and Prasad, they proved that " the Pythagorean orthogonality via $2-\mathrm{HH}$ norm is unique in any normed space" [9]. Besides that, they also define the Carlsson orthogonality via the 2$\mathrm{HH}$ norm in the paper [12].

For any $(x, y) \in X^{2}$, Kikianty and Dragomir defined the p-norm on $X^{2}$ as follows [10]:

$$
\|(x, y)\|_{p}= \begin{cases}\|x\|^{p}+\|y\|^{p}, & 1 \leq p<\infty \\ \max \{\|x\|,\|y\|\}, & p=\infty\end{cases}
$$

From (1), it is obvious that $\|(x, y)\|_{p}=\|(y, x)\|_{p}$, and therefore, the p-norm is symmetric. Using the concepts of Hermite-Hadamard's inequality, we have

$$
\int_{0}^{1}\|(1-t) x+t y\|^{p} d t \leq \frac{\|x\|^{p}+\|y\|^{p}}{2}=\frac{\|(x, y)\|_{p}^{p}}{2}<\infty .
$$

With the help of (2), they defined the p-HH norm on $X^{2}$ in the following ways [10]:

$$
\|(x, y)\|_{p-H H}= \begin{cases}\left(\int_{0}^{1}\|(1-t) x+t y\|^{p}\right) \frac{1}{p}, & \text { if } 1 \leq p<\infty \\ \sup _{t \in[0,1]}\|(1-t) x+t y\|, & \text { if } p=\infty .\end{cases}
$$

For all $x, y \in X$, it is obvious that $\|(x, y)\|_{p-H H}=\|(y, x)$ $\|_{p-H H}$. Therefore, the $\mathrm{p}-\mathrm{HH}$ norm is symmetric. They proved that $\left(X^{2},\|(.,)\|.\right)$ is a normed linear space because the nondegeneracy and homogeneity of the norm can be derived from (3) and the triangle inequality follows from Minkowski's inequality. If the norm on $X$ is induced by an inner product (., .), then as a special case of the p-HH norm, it is denoted by the $2-\mathrm{HH}$ norm. It is defined in the paper [9] as follows:

$$
\|(x, y)\|_{2-H H}=\int_{0}^{1}\|(1-t) x+t y\|^{2} d t .
$$

For any $p \geq 1$, the $\mathrm{p}$-norm and $\mathrm{p}-\mathrm{HH}$ norm are equivalent in $X^{2}$.

Definition 1. A real-valued function $f$ defined on a nonempty subset $X \subset \mathbb{R}^{n}$ is called convex if

(i) The domain $X$ of the function is convex

(ii) For any $x, y \in X$ and $\lambda \in[0,1]$

$$
f(\lambda x+(1-\lambda) y) \leq \lambda f(x)+(1-\lambda) f(y) .
$$

If the inequality $((5))$ is strict whenever $x \neq y$ and $0<\lambda$ $<1$, it is called strictly convex. To study the properties of orthogonality in normed linear space, it is interesting to investigate the following properties of orthogonality in ordinary Euclidean space as applied to normed linear space. For any Euclidean space $X$, let $x, y, z \in X$. Then, the following are considered as the main properties of orthogonality [9].

(i) Nondegeneracy: if $x \perp x$, then $x=0$.

(ii) Simplification: if $x \perp y$, then for any $\alpha \in \mathbb{R}, \alpha x \perp \alpha y$.

(iii) Continuity: if $\left\{x_{n}\right\},\left\{y_{n}\right\} \subset X$ such that $x_{n} \perp y_{n}$ for every $n \in \mathbb{N}, x_{n} \rightarrow x$ and $y_{n} \rightarrow y$, then $x \perp y$.

(iv) Homogeneity: if $x \perp y$, then $\alpha x \perp \beta y$ for all $\alpha, \beta \in \mathbb{R}$.

(v) Symmetry: if $x \perp y$, then $y \perp x$. 
(vi) Additivity: if $x \perp y$ and $x \perp z$, then $x \perp y+z$.

(vii) Existence: if $x \neq 0$, then there exist $\lambda \in \mathbb{R}$ such that $x \perp \lambda x+y$.

(viii) Uniqueness: for any $x \neq 0$, if there exists $\lambda \in \mathbb{R}$ such that $x \perp \lambda x+y$, then such $\lambda$ is unique.

In this paper, we mainly focus on the last property. Kapoor and Prasad proved that the Pythagorean orthogonality is unique in any normed linear space, but the isosceles orthogonality is unique if and only if the normed linear space is strictly convex [5]. Regarding the Robert orthogonality, the property of existence is satisfied only in an inner-product space [1].

Definition 2 [4]. A vector $x$ is said to be isosceles orthogonal to $y$ if and only if

$$
\|x-y\|=\|x+y\|
$$

Definition 3 [13]. A vector $x$ is said to be the Birkhoff-James orthogonal to $y$ if and only if

$$
\|x+\lambda y\| \geq\|x\| \text { for all } \lambda \in \mathbb{R} \text {. }
$$

\section{Main Result}

Definition 4 [11]. A vector $x$ is orthogonal to $y$ if

$$
\left\|x+\frac{1}{2} y\right\|^{2}+\left\|x-\frac{1}{2} y\right\|^{2}=\frac{1}{2}\|\sqrt{2} x+y\|^{2}+\|x\|^{2} .
$$

If the underlying space $X$ is a real inner product space and the relation (8) holds a. e on $[0,1]$. Then, using the concept of the 2-HH norm, we have

$$
\begin{gathered}
\int_{0}^{1}\left\|(1-t) x+\frac{1}{2} t y\right\|^{2} d t+\int_{0}^{1}\left\|(1-t) x-\frac{1}{2} t y\right\|^{2} d t \\
=\frac{1}{2} \int_{0}^{1}\|\sqrt{2}(1-t) x+t y\|^{2} d t \\
+\int_{0}^{1}\|(1-t) x\|^{2} d t .
\end{gathered}
$$

Now, the left-hand side of relation (9)

$$
\begin{aligned}
\int_{0}^{1} \|(1-t) x+ & \frac{1}{2} t y\left\|^{2} d t+\int_{0}^{1}\right\|(1-t) x-\frac{1}{2} t y \|^{2} d t \\
& =\int_{0}^{1}\left\langle(1-t) x+\frac{1}{2} t y,(1-t) x+\frac{1}{2} t y\right\rangle d t \\
+ & \int_{0}^{1}\left\langle(1-t) x-\frac{1}{2} t y,(1-t) x-\frac{1}{2} t y\right\rangle d t \\
& =\frac{1}{3}\|x\|^{2}+\frac{1}{12}\|y\|^{2}+\frac{1}{3}\|x\|^{2}+\frac{1}{12}\|y\|^{2} \\
& =\frac{2}{3}\|x\|^{2}+\frac{1}{6}\|y\|^{2} .
\end{aligned}
$$

Again, the right-hand side of relation (9)

$$
\begin{aligned}
\frac{1}{2} \int_{0}^{1}\|\sqrt{2}(1-t) x+t y\|^{2} d t+\int_{0}^{1}\|(1-t) x\|^{2} d t \\
=\frac{1}{2} \int_{0}^{1}\langle\sqrt{2}(1-t) x+t y, \sqrt{2}(1-t) x+t y\rangle d t \\
+\frac{1}{3}\|x\|^{2}=\frac{1}{2}\left(\frac{2}{3}\|x\|^{2}+\frac{1}{3}\|y\|^{2}\right)+\frac{1}{3}\|x\|^{2} \\
=\frac{2}{3}\|x\|^{2}+\frac{1}{6}\|y\|^{2} .
\end{aligned}
$$

Now, we consider a notion of orthogonality as follows: let $(X .\|\|$.$) be a normed space. A vector x \in X$ is said to be $2-\mathrm{HH}$ $\mathrm{N}$ orthogonal to $y \in X$ (denoted by $x \perp_{2-H H-N} y$ ) if and only if

$$
\int_{0}^{1}\left\|(1-t) x+\frac{1}{2} t y\right\|^{2} d t+\int_{0}^{1}\left\|(1-t) x-\frac{1}{2} t y\right\|^{2} d t=\frac{2}{3}\|x\|^{2}+\frac{1}{6}\|y\|^{2} .
$$

Kikianty and Dragomir in [9] proved that " the Pythagorean orthogonality via $2-\mathrm{HH}$ norm is unique in any normed space X". To prove this, they use the following lemma by omitting the proof. We give a detailed proof of the lemma as they stated in the paper [9].

Lemma 5. Let $x, y \in X$, where $X$ is the normed linear space. Let $h$ be a function on $\mathbb{R}$ defined by

$$
h(\mu):=\int_{0}^{1}\|(1-t) y+\mu(t x)\|^{2} d t
$$

Then, $h$ is a convex function on $\mathbb{R}$, and for any $r \in(0,1)$ and $\mu_{1}, \mu_{2} \in \mathbb{R}$ where $h\left(\mu_{1}\right) \neq h\left(\mu_{2}\right)$, we have

$$
h\left[r \mu_{1}+(1-r) \mu_{2}\right]<r h\left(\mu_{1}\right)+(1-r) h\left(\mu_{2}\right) .
$$

Proof. Let $r \in(0,1)$ and $\mu_{1}, \mu_{2} \in \mathbb{R}$ such that $h\left(\mu_{1}\right) \neq h\left(\mu_{2}\right)$. Then,

$$
\begin{aligned}
h\left[r \mu_{1}+(1-r) \mu_{2}\right]= & \int_{0}^{1}\left\|(1-t) y+\left[r \mu_{1}+(1-r) \mu_{2}\right](t x)\right\|^{2} d t \\
= & \int_{0}^{1}\left\|(1-t) y+r \mu_{1}(t x)+\mu_{2}(t x)-r \mu_{2}(t x)\right\|^{2} d t \\
= & \int_{0}^{1} \|(1-t) y+r \mu_{1}(t x)+\mu_{2}(t x)-r \mu_{2}(t x) \\
& -r(1-t) y+r(1-t) y \|^{2} d t \\
= & \int_{0}^{1} \| r\left[(1-t) y+\mu_{1}(t x)\right]+(1-r)[(1-t) y \\
& \left.+\mu_{2}(t x)\right]\left\|^{2} d t \leq \int_{0}^{1} r^{2}\right\|(1-t) y+\mu_{1}(t x) \|^{2} d t \\
& +(1-r)^{2} \int_{0}^{1}\left\|(1-t) y+\mu_{2}(t x)\right\|^{2} d t \\
& +2 r(1-r) \int_{0}^{1}\left\|(1-t) y+\mu_{1}(t x)\right\|\left\|(1-t) y+\mu_{2}(t x)\right\| d t
\end{aligned}
$$




$$
\begin{array}{rl}
=\int_{0}^{1} & r\left\|(1-t) y+\mu_{1}(t x)\right\|^{2} d t \\
& +(1-r) \int_{0}^{1}\left\|(1-t) y+\mu_{2}(t x)\right\|^{2} d t \\
& +\left(r^{2}-r\right) \int_{0}^{1}\left[\left\|(1-t) y+\mu_{1}(t x)\right\|^{2}+\|(1-t) y\right. \\
& +\mu_{2}(t x)\left\|^{2}-2\right\|(1-t) y+\mu_{1}(t x)\|\|(1-t) y \\
& \left.+\mu_{2}(t x) \|\right] d t=r h\left(\mu_{1}\right)+(1-r) h\left(\mu_{2}\right) \\
& -r(1-r) \int_{0}^{1}\left[\left\|(1-t) y+\mu_{1}(t x)\right\|-\|(1-t) y\right. \\
& \left.+\mu_{2}(t x) \|\right]^{2} d t \leq r h\left(\mu_{1}\right)+(1-r) h\left(\mu_{2}\right),
\end{array}
$$

which shows that $h$ is a convex function. Since $h\left(\mu_{1}\right) \neq$ $h\left(\mu_{2}\right)$, then the inequality will be strict and therefore

$$
h\left[r \mu_{1}+(1-r) \mu_{2}\right]<r h\left(\mu_{1}\right)+(1-r) h\left(\mu_{2}\right) .
$$

As a similar concept of Lemma 5, we also prove the following lemma which is useful to prove the uniqueness property of new orthogonality via the $2-\mathrm{HH}$ norm.

Lemma 6. Let $(X .\|\|$.$) be a normed space and x, y \in X$. Let $h$ be a function defined on $\mathbb{R}$ by

$$
h(\mu):=\int_{0}^{1}\left\|(1-t) y+\frac{\mu}{2}(t x)\right\|^{2} d t+\int_{0}^{1}\left\|(1-t) y-\frac{\mu}{2}(t x)\right\|^{2} d t .
$$

Then, $h$ is a convex function on $\mathbb{R}$ and for any $r \in(0,1)$, and $\mu_{1}, \mu_{2} \in \mathbb{R}$ where $h\left(\mu_{1}\right) \neq h\left(\mu_{2}\right)$, we have

$$
h\left[r \mu_{1}+(1-r) \mu_{2}\right]<r h\left(\mu_{1}\right)+(1-r) h\left(\mu_{2}\right) .
$$

Proof. Suppose $h(\mu)=f(\mu)+g(\mu)$, where

$$
\begin{aligned}
& f(\mu)=\int_{0}^{1}\left\|(1-t) y+\frac{\mu}{2}(t x)\right\|^{2} d t, \\
& g(\mu)=\int_{0}^{1}\left\|(1-t) y-\frac{\mu}{2}(t x)\right\|^{2} d t .
\end{aligned}
$$

First, we show that $f(k)$ is a convex function. Let $r \in(0,1)$ and $\mu_{1}, \mu_{2} \in \mathbb{R}$ such that $h\left(\mu_{1}\right) \neq h\left(\mu_{2}\right)$.

$$
\begin{aligned}
& f\left[r \mu_{1}+(1-r) \mu_{2}\right]=\int_{0}^{1}\left\|(1-t) y+\frac{1}{2}\left[r \mu_{1}+(1-r) \mu_{2}\right](t x)\right\|^{2} d t \\
& =\int_{0}^{1}\left\|(1-t) y+\frac{1}{2} r \mu_{1}(t x)+\frac{1}{2} \mu_{2}(t x)-\frac{1}{2} r \mu_{2}(t x)\right\|^{2} d t \\
& =\int_{0}^{1} \|(1-t) y+\frac{r \mu_{1}}{2}(t x)+\frac{\mu_{2}}{2}(t x)-r \frac{\mu_{2}}{2}(t x) \\
& +r(1-t) y-r(1-t) y \|^{2} d t \\
& =\int_{0}^{1} \| r\left[(1-t) y+\frac{\mu_{1}}{2}(t x)\right]+(1-r) \\
& \cdot\left[(1-t) y+\frac{\mu_{2}}{2}(t x)\right] \|^{2} d t \\
& \leq r^{2} \int_{0}^{1}\left\|(1-t) y+\frac{\mu_{1}}{2}(t x)\right\|^{2} d t \\
& +(1-r)^{2} \int_{0}^{1}\left\|(1-t) y+\frac{\mu_{2}}{2}(t x)\right\|^{2} d t \\
& +2 r(1-r) \int_{0}^{1}\left\|(1-t) y+\frac{\mu_{1}}{2}(t x)\right\| \|(1-t) y \\
& +\frac{\mu_{2}}{2}(t x)\left\|d t=r \int_{0}^{1}\right\|(1-t) y+\frac{\mu_{1}}{2}(t x) \|^{2} d t \\
& +(1-r) \int_{0}^{1}\left\|(1-t) y+\frac{\mu_{2}}{2}(t x)\right\|^{2} d t \\
& +r^{2} \int_{0}^{1}\left\|(1-t) y+\frac{\mu_{1}}{2}(t x)\right\|^{2} d t \\
& +(1-r)^{2} \int_{0}^{1}\left\|(1-t) y+\frac{\mu_{2}}{2}(t x)\right\|^{2} d t \\
& -r \int_{0}^{1}\left\|(1-t) y+\frac{\mu_{1}}{2}(t x)\right\|^{2} d t \\
& -(1-r) \int_{0}^{1}\left\|(1-t) y+\frac{\mu_{2}}{2}(t x)\right\|^{2} d t \\
& +2 r(1-r) \int_{0}^{1}\left\|(1-t) y+\frac{\mu_{1}}{2}(t x)\right\| \|(1-t) y \\
& +\frac{\mu_{2}}{2}(t x) \| d t=r f\left(\mu_{1}\right)+(1-r) f\left(\mu_{2}\right) \\
& +\left(r^{2}-r\right) \int_{0}^{1}\left[\left\|(1-t) y+\frac{\mu_{1}}{2}(t x)\right\|-\|(1-t) y\right. \\
& \left.+\frac{\mu_{1}}{2}(t x) \|\right]^{2} d t=r f\left(\mu_{1}\right)+(1-r) f\left(\mu_{2}\right) \\
& -\left(r-r^{2}\right) \int_{0}^{1}\left[\left\|(1-t) y+\frac{\mu_{1}}{2}(t x)\right\|-\|(1-t) y\right. \\
& \left.+\frac{\mu_{1}}{2}(t x) \|\right]^{2} d t \leq r f\left(\mu_{1}\right)+(1-r) f\left(\mu_{2}\right),
\end{aligned}
$$

which shows that $f$ is a convex function. Similarly, for the function

$$
g(\mu)=\int_{0}^{1}\left\|(1-t) t-\frac{\mu}{2}(t x)\right\|^{2} d t
$$

we can show that

$$
g\left[r \mu_{1}+(1-r) \mu_{2}\right] \leq r g\left(\mu_{1}\right)+(1-r) g\left(\mu_{2}\right),
$$

and we conclude that $g$ is also a convex function. Also, we know that the sum of two convex functions is also convex. Then, $h(\mu)=f(\mu)+g(\mu)$ is convex. Since $h\left(\mu_{1}\right) \neq h\left(\mu_{2}\right)$, then the inequality will be strict, and therefore 


$$
h\left[r \mu_{1}+(1-r) \mu_{2}\right]<r h\left(\mu_{1}\right)+(1-r) h\left(\mu_{2}\right) .
$$

Theorem 7. 2-HH-N orthogonality is unique in any normed space $X$.

Proof. The proof has a similar idea to that of Kapoor and Prasad (pp. 406) and Kikianty and Dragomir (pp. 41). Suppose $2-\mathrm{HH}-\mathrm{N}$ orthogonality is not unique. Then, we must have elements $x \neq 0$ and $y \in X$, and a $\lambda>0$ such that $x \perp_{2-H H-N} y$ and $x \perp_{2-H H-N} \lambda x+y$. Define a convex function

$$
h(\mu)=\int_{0}^{1}\left\|(1-t) y+\frac{\mu}{2}(t x)\right\|^{2} d t+\int_{0}^{1}\left\|(1-t) y-\frac{\mu}{2}(t x)\right\|^{2} d t .
$$

Now,

$$
\begin{aligned}
h(1) & =\int_{0}^{1}\left\|(1-t) y+\frac{1}{2}(t x)\right\|^{2} d t+\int_{0}^{1}\left\|(1-t) y-\frac{1}{2}(t x)\right\|^{2} d t \\
& =\frac{2}{3}\|y\|^{2}+\frac{1}{6}\|x\|^{2}=h(0)+\frac{1}{6}\|x\|^{2} .
\end{aligned}
$$

Setting $\beta=(2(1-t) \lambda) / t$ and note that

$$
\begin{aligned}
h(\beta)= & \int_{0}^{1}\left\|(1-t) y+\frac{1}{2} \cdot \frac{2(1-t) \lambda}{t}(t x)\right\|^{2} d t \\
& +\int_{0}^{1}\left\|(1-t) y-\frac{1}{2} \cdot \frac{2(1-t) \lambda}{t}(t x)\right\|^{2} d \\
= & \int_{0}^{1}\|(1-t) y+(1-t) \lambda x\|^{2} d t+\int_{0}^{1}\|(1-t) y-(1-t) \lambda x\|^{2} d t \\
= & \int_{0}^{1}\|(1-t)(y+\lambda x)\|^{2} d t+\int_{0}^{1}\|(1-t)(y-\lambda x)\|^{2} d t \\
= & \frac{\|y+\lambda x\|^{2}}{3}+\frac{\|y-\lambda x\|^{2}}{3},
\end{aligned}
$$

and

$$
\begin{aligned}
h(\beta+1)= & \int_{0}^{1}\left\|(1-t) y+\frac{1}{2} \cdot\left[\frac{2(1-t) \lambda}{t}+1\right](t x)\right\|^{2} d t \\
& +\int_{0}^{1}\left\|(1-t) y-\frac{1}{2} \cdot\left[\frac{2(1-t) \lambda}{t}+1\right](t x)\right\|^{2} d t \\
= & \int_{0}^{1}\left\|(1-t)(y+\lambda x)+\frac{t x}{2}\right\|^{2} d t+\int_{0}^{1} \|(1-t)(y-\lambda x) \\
& -\frac{t x}{2} \|^{2} d t=\frac{\|y+\lambda x\|^{2}}{3}+\frac{\|y-\lambda x\|^{2}}{3} \\
& +\frac{\|x\|^{2}}{6}=h(\beta)+\frac{\|x\|^{2}}{6} .
\end{aligned}
$$

Now, suppose that $0<\beta<1$ and note that $h(1) \neq h(0)$ (since $x \neq 0$ ), Lemma 6 gives

$$
h(\beta)<\beta h(1)+(1-\beta) h(0) .
$$

Also, $h(\beta+1) \neq h(\beta)$ (since $x \neq 0)$, and with the help of Lemma 6

$$
\begin{aligned}
h(1)< & \beta h(\beta)+(1-\beta) h(\beta+1)=\beta h(\beta)+(1-\beta) \\
& \cdot\left[h(\beta)+\frac{\|x\|^{2}}{6}\right]=\beta h(\beta)+(1-\beta) \\
& \cdot[h(\beta)+h(1)-h(0)] \Rightarrow h(\beta)>\beta h(1)+(1-\beta) h(0),
\end{aligned}
$$

which contradicts (28). Now, consider the case $\beta>1$, we have

$$
\begin{aligned}
h(1) \leq & \frac{\beta-1}{\beta} h(0)+\frac{1}{\beta} h(\beta)=h(0)+\frac{1}{\beta}[h(\beta)-h(0)] \Rightarrow h(1) \\
& -h(0) \leq \frac{1}{\beta}[h(\beta)-h(0)] \Rightarrow \frac{\|x\|^{2}}{6} \leq \frac{1}{\beta}[h(\beta)-h(0)] .
\end{aligned}
$$

Since $x \neq 0$, then, $h(\beta) \neq h(0)$, and using the Lemma 6, we have

$$
h(1)<\frac{\beta-1}{\beta} h(0)+\frac{1}{\beta} h(\beta) .
$$

Also, $h(1) \neq h(\beta+1)$ and Lemma 6 gives us

$$
\begin{aligned}
h(\beta)< & \frac{1}{\beta} h(1)+\frac{\beta-1}{\beta} h(\beta+1)=\frac{1}{\beta} h(1) \\
& \cdot+\frac{\beta-1}{\beta}[h(\beta)+h(1)-h(0)] \Rightarrow h(1) \\
> & \frac{1}{\beta} h(\beta)+\frac{\beta-1}{\beta} h(0),
\end{aligned}
$$

which contradicts the relation (31). For the case $\beta=1$, we have

$$
h(2)=h(1)+\frac{\|x\|^{2}}{6}=h(0)+\frac{\|x\|^{2}}{3} .
$$

This shows that $h(2) \neq h(0)$ (since $x \neq 0)$. Then, we have

$$
\begin{aligned}
h(1)< & \frac{1}{2} h(0)+\frac{1}{2} h(2)=\frac{1}{2}\left[h(0)+h(0)+\frac{\|x\|^{2}}{3}\right] \Rightarrow h(1) \\
& <h(0)+\frac{\|x\|^{2}}{6},
\end{aligned}
$$

which contradicts (25). Thus, in all cases, we get a contradiction. Hence, 2-HH-N orthogonality is unique in any normed space. 
In the following theorem, 2-HH- $\mathrm{R}$ and 2-HH-B denote the Robert orthogonality and Birkhoff-James orthogonality via the $2-\mathrm{HH}$ norm, respectively.

Theorem 8. Let $x, y \in X$, where $X$ is a real normed linear space equipped with an inner-product space over the field $\mathbb{K}=(\mathbb{R}$ or $\mathbb{C})$ and $\mu=\lambda t$. Then, 2-HH-R orthogonality implies 2$\mathrm{HH}-\mathrm{B}$ orthogonality and conversely.

Proof. Assume $x \perp_{2-H H-R} y$. Then, for any $\mu \in \mathbb{R}$,

$$
\begin{aligned}
\int_{0}^{1}\|(1-t) x+\mu y\|^{2} d t & =\int_{0}^{1}\|(1-t) x-\mu y\|^{2} d t \\
& =\int_{0}^{1}\langle(1-t) x-\mu y,(1-t) x-\mu y\rangle d t \\
& =\int_{0}^{1}\|(1-t) x\|^{2}-(1-t)\langle x, y\rangle \\
& -\mu(1-t)\langle y, x\rangle+\|\mu y\|^{2} d t \\
& =\int_{0}^{1}\|(1-t) x\|^{2} d t+\int_{0}^{1}\|\mu y\|^{2} d t \\
& \geq \int_{0}^{1}\|(1-t) x\|^{2} d t,
\end{aligned}
$$

which shows that $x$ is 2-HH-B orthogonal to $y$. To prove the converse part, it is enough to show that $x \perp_{2-H H-B} y \Rightarrow\langle x, y\rangle$ $=0 \Rightarrow x \perp_{2-H H-R} y$. Let $x \perp_{2-H H-B} y$. Then, for any $\lambda \in \mathbb{K}$,

$$
\begin{aligned}
\int_{0}^{1}\|(1-t) x+\lambda t y\|^{2} d t \geq & \int_{0}^{1}\|(1-t) x\|^{2} d t \Rightarrow \int_{0}^{1}\|(1-t) x\|^{2} d t \\
& +[\lambda\langle y, x\rangle+\overline{\lambda y, x}] \int_{0}^{1} t(1-t) d t \\
& +|\lambda|^{2}\|y\|^{2} \int_{0}^{1} t^{2} d t \geq \int_{0}^{1}\|(1-t) x\|^{2} d t \Rightarrow \operatorname{Re} \\
& \cdot[\lambda\langle y, x\rangle]+|\lambda|^{2}\|y\|^{2} \geq 0
\end{aligned}
$$

Now, for $\lambda=-\langle x, y\rangle / 2\|y\|^{2}$, inequality (36) becomes $|\langle y, x\rangle|^{2} / 4\|y\|^{2} \geq 0$. Therefore, we have $\langle x, y\rangle=0$. On the other hand, it is easy to show that $\langle x, y\rangle=0 \Rightarrow x \perp_{2-H H-R} y$.

\section{Conclusion}

We conclude that the new orthogonality via the 2-HH norm is unique in any normed linear space. Moreover, if the underlying space is a real inner product space, the Robert and Birkhoff orthogonality via the $2-\mathrm{HH}$ norm are equivalent.

\section{Data Availability}

There is no use of any data to support this study.

\section{Conflicts of Interest}

The authors declare that they do not have any conflict of interest for the publication of the article.

\section{References}

[1] G. Birkhoff, "Orthogonality and linear functionals in normed linear spaces," Transactions of the American Mathematical Society, vol. 61, no. 2, pp. 265-292, 1947.

[2] D. B. Robert, "On the geometry of abstract vector spaces," Tohoku Mathematical Journal, vol. 39, pp. 42-59, 1934.

[3] K. Paul, A. Mall, and D. Sain, "Orthogonality of bounded linear operators on complete Banach space," Annals of Mathematics, vol. 3, no. 3, pp. 669-709, 2018.

[4] R. C. James, "Orthogonality in normed linear spaces," Duke Mathematical Journal, vol. 12, no. 2, pp. 291-302, 1945.

[5] O. P. Kapoor and J. Prasad, "Orthogonality and characterizations of inner product spaces," Bulletin of the Australian Mathematical Society, vol. 19, no. 3, pp. 403-416, 1978.

[6] S. Carlsson, "Orthogonality in normed linear spaces," Arkiv för Matematik, vol. 1, no. 1, pp. 297-318, 1935.

[7] H. Martini and S. Wu, "On maps preserving isosceles orthogonality in normed linear spaces," Note di Matematica, vol. 29, no. 1, pp. 55-59, 2009.

[8] C. Alsina and M. S. Tomas, "On some orthogonality relations in real normed spaces and characterization of inner products," Bollettino dell'Unione Matematica Italiana, vol. 10, no. 8, pp. 513-520, 2007.

[9] S. S. Dragomir and E. Kikianty, "Hermite-hadamard's inequality and the p-hh-norm on the cartesian product of two copies of a normed space," Mathematical Inequalities and Applications, vol. 13, no. 1, pp. 1-32, 1935.

[10] E. Kikianty and S. S. Dragomir, "Orthogonality connected with integral means and characterizations of inner product spaces," Journal of Geometry, vol. 98, no. 10, pp. 33-49, 2010.

[11] P. M. Bajracharya and B. P. Ojha, "Birkhoff orthogonality and different particular cases of Carlsson's orthogonality on normed linear Spaces," Journal of Mathematics and Statistics, vol. 16, no. 1, pp. 133-141, 2020.

[12] E. Kikianty and S. S. Dragomir, "On Carlsson type orthogonality and characterization of inner product space," Filomat, vol. 26, no. 4, pp. 859-870, 2012.

[13] G. Birkhoff, "Orthogonality in linear metric spaces," Duke Mathematical Journal, vol. 1, no. 2, pp. 169-172, 1935. 